\title{
The Results of Adductor Magnus Tenodesis in Adolescents with Recurrent Patellar Dislocation
}

\author{
Krzysztof Malecki, ${ }^{1}$ Jaroslaw Fabis, ${ }^{2}$ Pawel Flont, ${ }^{1}$ and Kryspin Ryszard Niedzielski ${ }^{1}$ \\ ${ }^{1}$ Clinic of Orthopaedics and Traumatology, Polish Mother's Memorial Hospital Research Institute, Rzgowska 281/289, \\ 93-338 Lodz, Poland \\ ${ }^{2}$ Department of Arthroscopy, Minimally Invasive Surgery and Sports Traumatology, Medical University of Lodz, \\ Żeromskiego 113, 90-549 Lodz, Poland
}

Correspondence should be addressed to Pawel Flont; flont2002@yahoo.com

Received 28 September 2014; Revised 25 January 2015; Accepted 29 January 2015

Academic Editor: Konstantinos Anagnostakos

Copyright (c) 2015 Krzysztof Malecki et al. This is an open access article distributed under the Creative Commons Attribution License, which permits unrestricted use, distribution, and reproduction in any medium, provided the original work is properly cited.

Recurrent dislocation of the patella is a common orthopaedic problem which occurs in about $44 \%$ of cases after first-time dislocation. In most cases of first-time patellar dislocation, the medial patellofemoral ligament (MPFL) becomes damaged. Between 2010 and 2012, 33 children and adolescents (39 knees) with recurrent patellar dislocation were treated with MPFL reconstruction using the adductor magnus tendon. The aim of our study is to assess the effectiveness of this surgical procedure. The outcomes were evaluated functionally (Lysholm knee scale, the Kujala Anterior Knee Pain Scale, and isokinetic examination) and radiographically (Caton index, sulcus angle, congruence angle, and patellofemoral angle). Four patients demonstrated redislocation with MPFL graft failure, despite the fact that patellar tracking was found to be normal before the injury, and the patients had not reported any symptoms. Statistically significant improvements in Lysholm and Kujala scales, in patellofemoral and congruence angle, were seen $(P<0.001)$. A statistically significant improvement in the peak torque of the quadriceps muscle and flexor was observed for $60^{\circ} / \mathrm{sec}$ and $180^{\circ} / \mathrm{sec}$ angular velocities $(P=0.01)$. Our results confirm the efficacy of MPFL reconstruction using the adductor magnus tendon in children and adolescents with recurrent patellar dislocation.

\section{Introduction}

Recurrent dislocation of the patella is a common orthopaedic problem which occurs in about $44 \%$ of cases after firsttime dislocation [1]. Several dozen descriptions of surgical methods for recurrent patellar dislocation are given in the literature, and an appropriate one, such as proximal, distal realignment or sulcus plasty [2-5], must be selected depending on the severity of anatomical and functional problems, as well as the age of the patient. In most cases of firsttime patellar dislocation, the medial patellofemoral ligament (MPFL), which is the most important stabiliser within the first 30 degrees of flexion, becomes damaged [6]. However, the MPFL may be reconstructed to realign a patella with deficient proximal medial restraints, a procedure which can be combined with lateral retinacular release, or a distal realignment technique such as tibial tuberosity osteotomy or patellar tendon partial transposition, in skeletal-immature patients experiencing patellar tilt or shift [2,7-10].

An additional test rarely used in the diagnosis and treatment of recurrent patellar dislocation is based on isokinetic measurements. Isokinetic evaluation performed after surgery allows the rehabilitation programme to be optimised and is intended to return the muscle strength of the extensor system to a pretreatment status comparable to that of the healthy side. Testing the strength of the muscle is important because the vastus medialis oblique (VMO) serves to control patellar tracking through varying degrees of knee motion and prevents the occurrence of anterior knee pain [11]. In patellar instability, it is critical to maintain the dynamic balance of the quadriceps to limit the dominance of the lateral structures. Isokinetic testing evaluates the dynamic relationships during 
TABLE 1: Demographic data and clinical outcomes according to the Lysholm, Kujala, and Beighton scales.

\begin{tabular}{lcccc}
\hline & $X$ & Range & SD & $\mathrm{Me}$ \\
\hline $\begin{array}{l}\text { Age at the time of } \\
\text { surgery (years) }\end{array}$ & 16 & $8-18$ & 2.7 & 17 \\
$\begin{array}{l}\text { Follow-up (years) } \\
\begin{array}{l}\text { Lysholm scale } \\
\text { pre-/postoperation }\end{array}\end{array}$ & 2.6 & $2-3$ & 0.5 & 3 \\
$\begin{array}{l}\text { Kujala scale } \\
\text { pre-/postoperation }\end{array}$ & $66 / 92$ & $30-95 / 59-100$ & $14 / 11$ & $62 / 95$ \\
\begin{tabular}{l} 
Beighton scale \\
\hline
\end{tabular} & 4.3 & $0-80 / 70-100$ & $11 / 9$ & $68 / 95$ \\
\end{tabular}

knee movements, and, as it is reproducible and fully objective, it may act as one of the endpoints in the study of patellar instability treatment methods [12-15].

\section{Materials and Methods}

Between 2010 and 2012, 33 patients (39 knees) with recurrent patellar dislocation were treated with MPFL reconstruction using the adductor magnus tendon. The average age at the time of surgery was 16 years (range: 8 to 18 years, SD 2.5). All patients (20 girls and 13 boys) were available for followup examination and were analysed prospectively. The mean follow-up was 2.6 years (range: 2 to 3 years, SD 0.5) (Table 1).

In the case of 9 knees with patellar shift and lateralisation of the tibial tuberosity, normal patellar tracking was restored by supplementing the MPFL reconstruction with the transfer of one half of the patellar tendon. In the case of $23 \mathrm{knees}$ with patellar tilt, lateral retinacular release was performed. The following study inclusion criteria applied: patellar dislocation that had occurred at least twice, positive apprehension sign, completed research protocol, and age under 18 years at the time of surgery. Subjects with first-time patellar dislocation or habitual patellar dislocation, as well as patients with osteochondral fracture and those with a history of knee surgery, were excluded. Of the 39 knees which met the study inclusion criteria, 14 had experienced two dislocations, 16 three dislocations, and 9 multiple dislocations (from 4 to 12). The trochlea dysplasia and patella alta did not influence the treatment protocol.

The outcomes were evaluated functionally and radiographically. The number of recurrent dislocations was noted, and positive apprehension tests were carried out. Laxity analysis based on the Beighton scale was also performed: a score of $\geq 4$ being considered as ligamentous laxity [16]. The evaluation also included patient-reported outcome measures (Lysholm knee scale and the Kujala Anterior Knee Pain Scale) and isokinetic examination [17, 18]. Quadriceps and hamstring maximal peak torque at 60 and $180^{\circ} / \mathrm{sec}$ were measured 1 month before and 1 year after surgery (Biodex Multi-Joint System-Pro, Biodex Medical Systems, Inc., New York, USA).

The radiological assessment before and 1 year after surgery included a lateral standing weight-bearing position and axial X-ray examination according to Merchant at 45

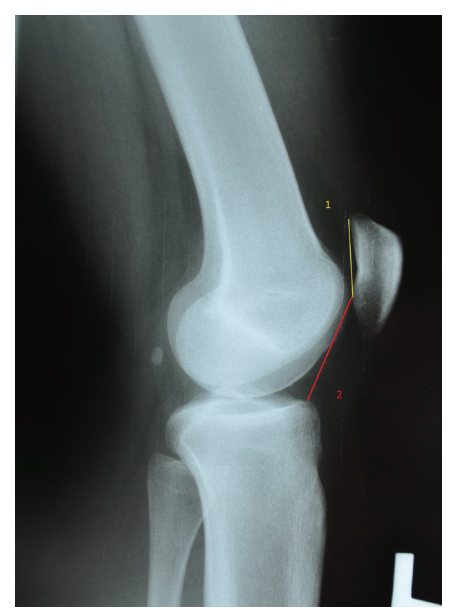

FIGURE 1: Example of Caton index measurement in lateral standing view. Caton index $=\mathrm{L} 2 / \mathrm{L} 1$.

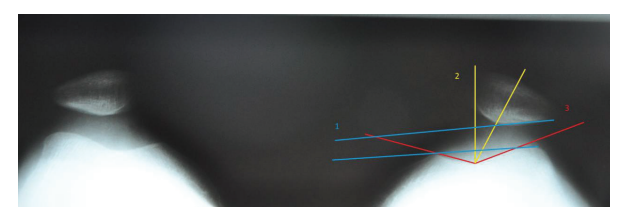

Figure 2: Preoperative X-rays according to Merchant view in $45^{\circ}$ of flexion with quadriceps tension. (1) Patellofemoral angle. (2) Congruence angle. (3) Sulcus angle.

degrees of knee flexion. The following parameters were assessed: Caton index, sulcus angle, congruence angle, and patellofemoral angle. Patellar height was determined by lateral projection $\mathrm{X}$-ray performed under full loading according to Caton (Figure 1), and sulcus angle, congruence angle, and patellofemoral angle were assessed based on axial radiograms obtained by Merchant view [4, 5, 14] (Figures 2 and 3). The following values were adopted as normal: sulcus angle $\leq 147^{\circ}$, patellar height $\leq 1.2$, patellofemoral angle being open laterally (lower than $0^{\circ}$ ), and a congruence angle from $-17^{\circ}$ to $5^{\circ}$, with values greater than $5^{\circ}$ implying patellar lateralisation [19-25].

\section{Surgical Technique}

All patients were operated on by the first author according to the technique first described by Avikainen from 6 weeks to 12 weeks after last injury [26]. The aim of the rehabilitation program performed before surgery was to achieve full range of motion of the knee without effusion. A medial incision approximately $10-15 \mathrm{~cm}$ long was performed (Figure 4(a)). The deep fascia and retinacula were incised and the vastus medialis muscle was elevated anteriorly (Figure 4(b)), following which the adductor magnus tendon was dissected and cut from the musculotendinous junction, while the distal insertion was left intact (Figure 4(c)). A tunnel was created in the patella (Figures 4(d) and 4(e)), through which the tendon was sutured with Vicryl 1.0 at an appropriate tension with the knee flexed at $30^{\circ}$ (Figure $4(\mathrm{f})$ ). The graft was passed between the synovium and the fibrous membrane of the articular 


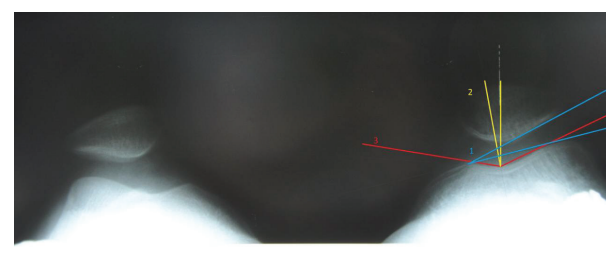

Figure 3: Postoperative X-rays according to Merchant view at $45^{\circ}$ of flexion with quadriceps tension. (1) Patellofemoral angle. (2) Congruence angle. (3) Sulcus angle.

capsule. From the same incision, a lateral retinacular release was performed in the case of patellar tilt. Lateral release that was performed if the patellofemoral angle was found to be open medially in Merchant view X-rays and patellar tilt was present on physical examination. Tilt was assessed by palpating the medial and lateral borders of the patella with the knee extended or slightly flexed, as described by Kolowich et al. [27].

In nine of the knees, partial patellar medial transposition according to Roux-Goldthwait with resuturing under the periosteum medial to the tibial tuberosity was performed $[21,28]$. Distal realignment was performed in the case of the $Q$ angle being greater than $20^{\circ}$ and the presence of a patellar shift with an increased congruence angle. According to Merchant, the normal value of congruence angle ranges from $5^{\circ}$ to $-17^{\circ}$. Orthosis immobilisation was used for 6 weeks. For the first 2 weeks, the orthosis was completely locked in a 10degree flexion; for the next 2 weeks, a 0 -30-degree range of motion was permitted in the orthosis, increasing to 0-60 degrees after another 2 weeks. The full loading of the operated limb was allowed 4 weeks after the procedure. Outpatient rehabilitation was carried out for 6 months.

The nonparametric Wilcoxon test for related data and Mann-Whitney $U$ test for unrelated data were used to compare the frequencies of significant deficits, as was the independent $\chi^{2}$ test for a four-field array. Statistical significance was assumed for $P<0.05$. The analysis was performed using the STATISTICA software package, ver. 10 (Statsoft, Inc. 2011, DASS, http://www.statsoft.com/). The study was approved by our institutional review board (approval issue date October 23, 2010).

\section{Results}

Research protocol was completed in all patients. Recurrent dislocation during follow-up was reported in 4 patients during the 2- to 3-year period following surgery (4 knees, $10.3 \%)$ : during sports in three cases and while dancing in one case. These patients experienced intra-articular haematoma following the recurrent dislocation, and ultrasound demonstrated signs of MPFL avulsion tear $(n=2)$ or rupture of the ligament $(n=2)$. The recurrent events occurred in patients without partial transposition of the patellar tendon.

While a positive apprehension test was seen in all patients at screening, it was positive only in 7 cases $(17.9 \%)$ at follow-up; this number includes all patients with recurrent dislocation. All patients in the present study achieved the full range of knee motion and none reported excessive pressure of the patella. Twenty-one patients met the diagnostic criterion for ligamentous laxity $(63.6 \%)$. The mean value in this scale for the entire cohort was 4.3 (range of 0 to 8 ). Statistically significant improvements were achieved according to both the Lysholm and Kujala scales $(P<0.001)$. The mean presurgery score was 64 points (ranging from 30 to 95 points), increasing to 91 points at follow-up (59 to 100 points) according to the Lysholm scale, while the mean presurgery score was 66 points ( 38 to 88 points) increasing to 92 points at follow-up (70 to 100 points) according to the Kujala scale (Table 1).

\section{Imaging}

Statistically significant improvements in patellofemoral and congruence angle were seen $(P<0.001)$. Patellar tilt was observed in 25 knees preoperatively and in 7 after surgery, whereas patellar shift was found in 32 knees preoperatively and in 10 knees after surgery. No statistically significant improvement was observed in sulcus angle $(P=0.07)$ or Caton index $(P=0.614)$, and no improvement was found in any of the 28 knees with abnormal sulcus angle. Similarly, while the Caton index was normalised in 7 knees, it remained abnormal in 16 knees (Table 2).

\section{Isokinetic Evaluation}

A statistically significant improvement in the peak torque of the quadriceps muscle was observed for both tested angular velocities $(P=0.01)$. Improvement was seen in 24 operated limbs when measured at a speed of $60^{\circ} / \mathrm{sec}$ and in 26 operated limbs at $180^{\circ} / \mathrm{sec}$. A deficit of peak torque of more than $10 \%$ relative to the opposite limb after surgery was found in 24 limbs when tested at $60^{\circ} / \mathrm{sec}$ and in $20 \mathrm{limbs}$ at $180^{\circ} / \mathrm{sec}$.

As with the quadriceps examination, the isokinetic evaluation of the flexor revealed a statistically significant increase of peak torque for both angular velocities after treatment $(P<0.001)$. Progress was noted in 30 knees at 60 degrees $/ \mathrm{sec}$ and in 34 knees at a speed of $180^{\circ} / \mathrm{sec}$. A peak torque deficit of more than $10 \%$ relative to the opposite limb in the flexors after surgery was found in 10 limbs when tested at $60^{\circ} / \mathrm{sec}$ and in 14 limbs at $180^{\circ} / \mathrm{sec}$ (Table 3 ).

\section{Discussion}

The most important finding in the present study was that the described surgical technique is efficacious in prevention of recurrent patellar dislocation. Four patients $(10.3 \%)$ demonstrated recurrence with MPFL graft failure, despite the fact that patellar tracking was found to be normal before the injury causing redislocation, and the patients had not reported any symptoms.

A statistically significant improvement in patientreported outcome according to the Lysholm and Kujala scale was observed throughout the entire cohort, which confirms the effectiveness of MPFL reconstruction. These findings are supported by the results of the radiological examination, which showed improvement in the two most important 


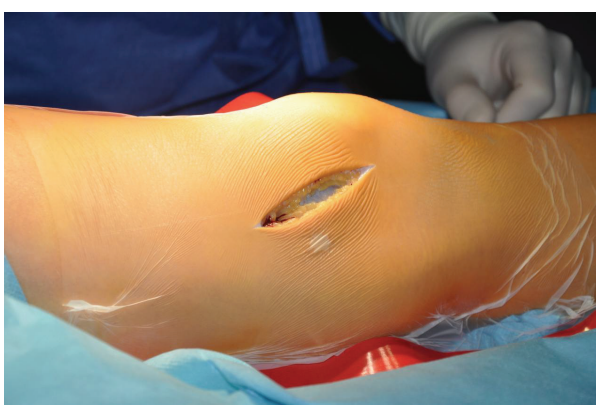

(a)

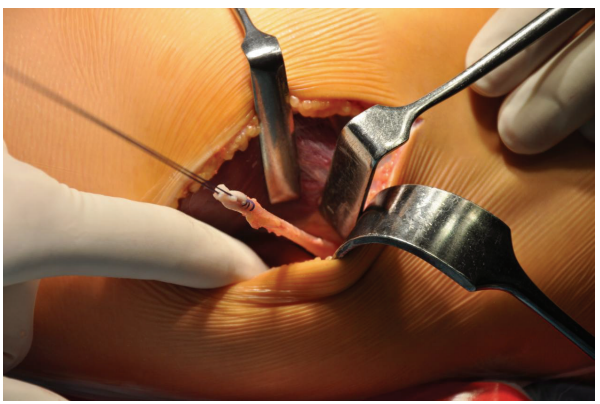

(c)

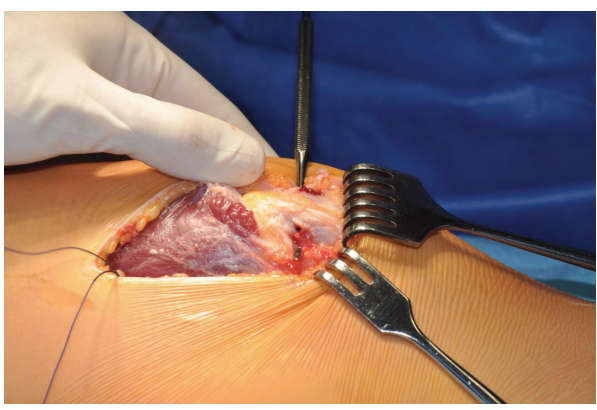

(e)

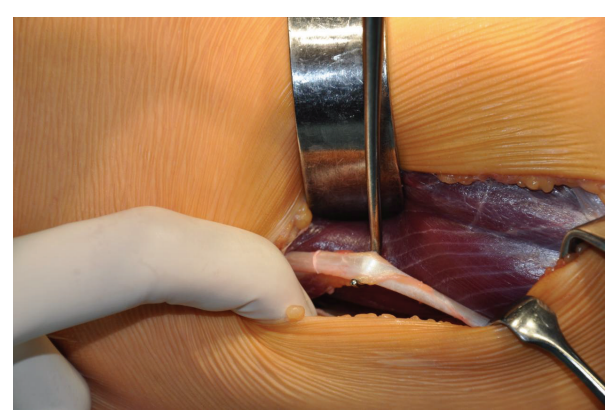

(b)

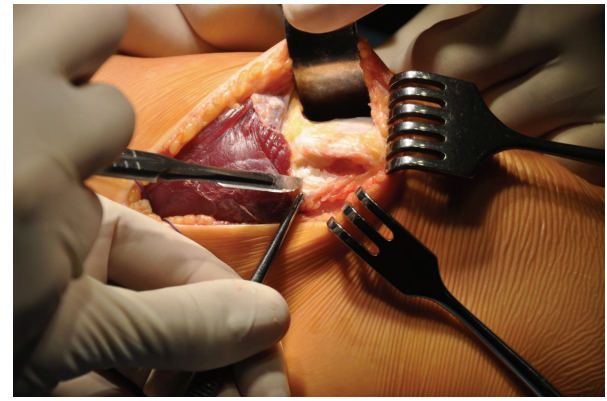

(d)

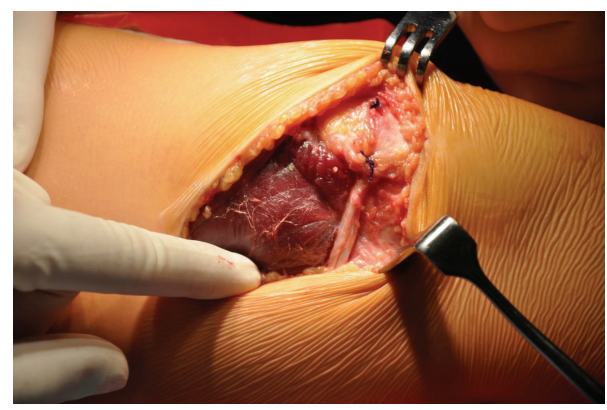

(f)

Figure 4: Surgical technique. (a) Incision. (b) Harvesting of the adductor magnus tendon lying under the vastus medialis. (c) Adductor magnus cut from its proximal insertion and suture with 1.0 Vicryl. (d) Preparation of the superomedial corner of the patellae. The knives show MPFL insertion to the patellae. (e) The patellae were drilled from the medial to lateral border. Drilling was started at the point where MPFL is inserted. (f) The adductor magnus tendon was passed through the patellae and sutured with 1.0 Vicryl with appropriate tension, with the knee flexed at $30^{\circ}$.

TABLE 2: Radiological outcomes in screening and check-up.

\begin{tabular}{|c|c|c|c|c|c|c|c|c|c|}
\hline & \multicolumn{2}{|c|}{$\begin{array}{c}X \\
\mathrm{Me}\end{array}$} & \multicolumn{2}{|c|}{$\begin{array}{c}\text { SD } \\
\text { range }\end{array}$} & \multicolumn{2}{|c|}{ Normal values $(n)$} & \multicolumn{2}{|c|}{ Abnormal values $(n)$} & \multirow[t]{2}{*}{$P$ value } \\
\hline & Pre & Post & Pre & Post & Pre & Post & Pre & Post & \\
\hline Sulcus angle $\left[{ }^{\circ}\right]$ & $\begin{array}{l}149 \\
148\end{array}$ & $\begin{array}{l}148 \\
150\end{array}$ & $\begin{array}{c}6.8 \\
135-164\end{array}$ & $\begin{array}{c}7.1 \\
130-160\end{array}$ & 11 & 11 & 28 & 28 & $P=0.801$ \\
\hline Caton index & $\begin{array}{l}1.27 \\
1.28\end{array}$ & $\begin{array}{l}1.24 \\
1.14\end{array}$ & $\begin{array}{c}0.26 \\
0.77-2\end{array}$ & $\begin{array}{c}0.3 \\
0.93-2\end{array}$ & 16 & 23 & 23 & 16 & $P=0.117$ \\
\hline Patellofemoral angle $\left[{ }^{\circ}\right]$ & $\begin{array}{l}4 \\
2\end{array}$ & $\begin{array}{l}-4 \\
-5\end{array}$ & $\begin{array}{c}10 \\
-10-34\end{array}$ & $\begin{array}{c}5 \\
-15-8\end{array}$ & 14 & 32 & 25 & 7 & $P<0.001$ \\
\hline Congruence angle $\left[{ }^{\circ}\right]$ & $\begin{array}{l}21 \\
17\end{array}$ & $\begin{array}{l}-5 \\
-2\end{array}$ & $\begin{array}{c}20 \\
-15-75\end{array}$ & $\begin{array}{c}12 \\
-30-20\end{array}$ & 7 & 29 & 32 & 10 & $P<0.001$ \\
\hline
\end{tabular}


TABLE 3: Data for isokinetic measurement during screening and check-up.

\begin{tabular}{lcccccc}
\hline & & Progress of peak torque $(n=39)$ & \multicolumn{3}{c}{ Peak torque deficit $>10 \%(n=39)$} \\
& & No & $P$ value & Screening & check-up & $P$ value \\
\hline Maximal quadriceps & At $60^{\circ} / \mathrm{s}$ & 24 & $P=0.010$ & 19 & 24 & $P=0.255$ \\
muscle torque values & At $180^{\circ} / \mathrm{s}$ & 26 & $P=0.010$ & 16 & 20 & $P=0.346$ \\
\hline \multirow{2}{*}{$\begin{array}{l}\text { Maximal knee flexor } \\
\text { muscle torque values }\end{array}$} & At $60^{\circ} / \mathrm{s}$ & 30 & $P=0.001$ & 15 & 10 & $P=0.027$ \\
& At $180^{\circ} / \mathrm{s}$ & 34 & $P=0.001$ & 13 & 14 & $P=0.811$ \\
\hline
\end{tabular}

indicators of the patellar position: the congruence angle and the patellofemoral angle.

Avikainen et al. observed a recurrent dislocation in 1 of 14 patients examined in a 7-year follow-up period after adductor magnus tenodesis; however, the inclusion criteria were different than those used in the present study, because first-time dislocations were included [26]. Another study by Sillanpää et al. compares the effectiveness of MPFL reconstruction with that of distal realignment in treating recurrent patellar dislocation [29]. The study group included 15 patients with MPFL reconstruction from the tendon of the adductor magnus muscle, and 21 patients after a Roux-Goldthwait operation. The MPFL group included 10 good or very good outcomes, 5 satisfactory and poor outcomes, and 1 patient with a recurrent dislocation. Similarly, in the Roux-Goldthwait group, there were 12 good and very good outcomes, 9 poor and satisfactory outcomes, and 3 cases of recurrent dislocation. Follow-up Xray scans revealed no degenerative lesions in the MPFL group but 5 cases in the Roux-Goldthwait group.

Panagopoulos describes a technique of MPFL reconstruction using a semitendinosus tendon which is passed through the medial intermuscular septum at the adductor magnus insertion. This graft, where the intermuscular septum acts as a pulley for the tendon, functions similar to the reconstructed MPFL used in our study. In short follow-up of 25 patients, no cases of redislocation were recorded and one patient sustained a patellar facture. The author reports improvement in IKDC, Tegner, Lysholm, and Kujala scores [30].

In 2009, Sillanpää et al. published a minimally invasive method of tendon collection, which resulted in a reduction in wound length [31]. However, in the authors' experience, due to the vascular topography of anatomical variants of vessels, the preferred method for tendon harvest should be the open method. It allows simultaneous lateral retinacular release from the same incision if necessary. If arthroscopy is performed before MPFL reconstruction, lateral retinaculum release is carried out with a coagulation device which is placed in the inferolateral portal. Arthroscopy is also useful for diagnosis and treatment of the cartilage lesion at the medial patellar facet and the lateral edge of the femoral trochlea.

Although a comprehensive literature review reveals no other descriptions of this specific surgical procedure, many other methods for MPFL tendon reconstruction with free grafts from the gracilis, semitendinosus, quadriceps tendon, fascia lata, or iliotibial band have been reported. The recurrences of dislocation observed with various grafts are similar and range from 5 to $10 \%$ [32-38].

The findings of the present study do not reveal any improvements of patellar height ratio or sulcus angle after surgery. In principle, no such changes were to be expected with this method, as trochlear rebuilding is only theoretically possible in young patients with long-term follow-up, and it should not be expected in the adolescent group [39, 40]. Another possible concern is based on the nonanatomical location of the graft and its adverse impact on the change in patellar height; however, as the average Caton index was found to decrease slightly, no such relationship was confirmed; as a result, the indicator was found to be normalised in more patients at the follow-up assessment. The femoral insertion on the adductor tubercle is very close to the native femoral MPFL attachment and in the anatomic study ranges from $6 \mathrm{~mm}$ to $15 \mathrm{~mm}$, mean $11 \mathrm{~mm}$ [41]. In different cadaveric study the medial patellofemoral ligament attachment on the femur was $1.9 \mathrm{~mm}$ anterior and $3.8 \mathrm{~mm}$ distal to the adductor tubercle [42].

The isokinetic analysis showed a significant improvement in peak torque of the quadriceps and flexors for both speeds evaluated. It should be noted, however, that a clinically significant deficit of greater than $10 \%$ remained, compared to the opposite side, which may lead to an increased risk of injury [14]. Treatment and rehabilitation after surgery significantly decreased the number of deficits in relation to the opposite side only in the flexor examination at 60 degrees/s. Hence, there is a need for a comparison of isokinetic testing between the two sides to be incorporated in any planned rehabilitation programme aimed at regaining extensor muscle strength.

A prospective study of 20 patients evaluated before and after a procedure for recurrent patellar dislocation based on isokinetic evaluation at speeds of 6,12 , and $60^{\circ} / \mathrm{sec}$ was published by Rauschning et al. In a 20-month follow-up period, the authors reported a slight, nonsignificant improvement in the torque values of the quadriceps muscle, as well as a significant decrease in performance compared with healthy volunteers from a control group [43]. Similar to the present study, Ronga et al. report persistent significant weakness in the operated limbs of 28 patients with recurrent patellar dislocation, compared to the opposite limbs, at the last follow-up assessment, with a mean follow-up of 3.1 years [12]. Similarly, in a prospective study involving 25 sport-practising skeletally immature patients with recurrent patellar dislocation, Oliva et al. reported a decrease in the muscular 
parameters in the operated limbs after an average period after surgery of 3.8 years [13].

The procedure described by Avikainen allows autologous, vascularised graft to be used in growing patients with open growth cartilages. As most other methods of MPFL reconstruction involve the risk of damage to the growth cartilage when fixing the graft, these procedures are mainly performed in adults. However, the perceived disadvantages of this method include the slightly nonanatomical location of the graft and the risk of damage to the femoral vessels, the saphenous branch of the descending genicular artery and the saphenous nerve which lie close by [41, 42]. An analysis of surgical material indicates the most significant limitations of this method to be the lack of tendon in some cases and its insufficient length or small cross section, all of which make reconstruction impossible. In the present study, no tendon was found intraoperatively in two knees, and the graft was too short in two others; these patients were not included in the study.

\section{Conclusions}

Our results confirm the efficacy of MPFL reconstruction using the adductor magnus tendon in children and adolescents with recurrent patellar dislocation. In the presence of a deficit in quadriceps and flexor strength, patients may benefit from isokinetic analysis when planning the postoperative rehabilitation programme following MPFL reconstruction with an adductor magnus tendon.

\section{Conflict of Interests}

The authors declare that there is no conflict of interests regarding the publication of this paper.

\section{Acknowledgment}

This work was sponsored by the Polish Ministry of Science \& Higher Education, Polish Mother's Memorial Hospital Research Institute Young Researcher Internal Grant no. 2012/59-MN.

\section{References}

[1] R. H. Cofield and R. S. Bryan, "Acute dislocation of the patella: results of conservative treatment," Journal of Trauma, vol. 17, no. 7, pp. 526-531, 1977.

[2] D. C. Fithian, E. W. Paxton, and A. B. Cohen, "Indications in the treatment of patellar instability," The Journal of Knee Surgery, vol. 17, no. 1, pp. 47-56, 2004.

[3] H. Mäenpää, H. Huhtala, and M. U. K. Lehto, "Recurrence after patellar dislocation. Redislocation in 37/75 patients followed for 6-24 years," Acta Orthopaedica Scandinavica, vol. 68, no. 5, pp. 424-426, 1997.

[4] A. C. Colvin and R. V. West, "Patellar instability," The Journal of Bone and Joint Surgery-American Volume, vol. 90, no. 12, pp. 2751-2762, 2008.

[5] Z. Dannawi, V. Khanduja, C. R. Palmer, and M. El-Zebdeh, "Evaluation of the modified Elmslie-Trillat procedure for patellofemoral dysfunction," Orthopedics, vol. 33, no. 1, article 13, 2010.

[6] P. I. Sallay, J. Poggi, K. P. Speer, and W. E. Garrett, "Acute dislocation of the patella: a correlative pathoanatomic study," American Journal of Sports Medicine, vol. 24, no. 1, pp. 52-60, 1996.

[7] M. Lind, B. W. Jakobsen, B. Lund, and S. E. Christiansen, "Reconstruction of the medial patellofemoral ligament for treatment of patellar instability," Acta Orthopaedica, vol. 79, no. 3, pp. 354-360, 2008.

[8] E. B. Crosby and J. Insall, "Recurrent dislocation of the patella. Relation of treatment to osteoarthritis," The Journal of Bone and Joint Surgery-American Volume, vol. 58, no. 1, pp. 9-13, 1976.

[9] F. Wang, H.-J. Kang, B.-C. Chen, W. Chen, Y.-L. Su, and Y.-Z. Zhang, "Combination of medial patellofemoral ligament reconstruction with vastus medialis advancement for chronic patellar dislocation," Chinese Medical Journal, vol. 123, no. 21, pp. 3024$3029,2010$.

[10] P. Beck, N. A. T. Brown, P. E. Greis, and R. T. Burks, "Patellofemoral contact pressures and lateral patellar translation after medial patellofemoral ligament reconstruction," American Journal of Sports Medicine, vol. 35, no. 9, pp. 1557-1563, 2007.

[11] F. J. Lieb and J. Perry, "Quadriceps function. An anatomical and mechanical study using amputated limbs," The Journal of Bone and Joint Surgery-American Volume, vol. 50, no. 8, pp. 1535$1548,1968$.

[12] M. Ronga, F. Oliva, U. G. Longo, V. Testa, G. Capasso, and N. Maffulli, "Isolated medial patellofemoral ligament reconstruction for recurrent patellar dislocation," The American Journal of Sports Medicine, vol. 37, no. 9, pp. 1735-1742, 2009.

[13] F. Oliva, M. Ronga, U. G. Longo, V. Testa, G. Capasso, and N. Maffulli, "The 3-in-1 procedure for recurrent dislocation of the patella in skeletally immature children and adolescents," The American Journal of Sports Medicine, vol. 37, no. 9, pp. 1814-1820, 2009.

[14] T. E. Hewett, G. D. Myer, and B. T. Zazulak, "Hamstrings to quadriceps peak torque ratios diverge between sexes with increasing isokinetic angular velocity," Journal of Science and Medicine in Sport, vol. 11, no. 5, pp. 452-459, 2008.

[15] J. Fabiś, "The impact of isokinetic training program on the peak torque of the quadriceps and knee flexors after anterior cruciate ligament reconstruction with hamstrings," Ortopedia Traumatologia Rehabilitacja, vol. 9, no. 5, pp. 527-531, 2007.

[16] P. Beighton, L. Solomon, and C. L. Soskolne, "Articular mobility in an African population," Annals of the Rheumatic Diseases, vol. 32, no. 5, pp. 413-418, 1973.

[17] U. M. Kujala, L. H. Jaakkola, S. K. Koskinen, S. Taimela, M. Hurme, and O. Nelimarkka, "Scoring of patellofemoral disorders," Arthroscopy, vol. 9, no. 2, pp. 159-163, 1993.

[18] Y. Tegner and J. Lysholm, "Rating systems in the evaluation of knee ligament injuries," Clinical Orthopaedics and Related Research, vol. 198, pp. 43-49, 1985.

[19] J. S. Mulford, C. J. Wakeley, and J. D. J. Eldridge, "Assessment and management of chronic patellofemoral instability," The Journal of Bone and Joint Surgery-British Volume, vol. 89, no. 6, pp. 709-716, 2007.

[20] A. C. Merchant, R. L. Mercer, R. H. Jacobsen, and C. R. Cool, "Roentgenographic analysis of patellofemoral congruence," The Journal of Bone and Joint Surgery-American Volume, vol. 56, no. 7, pp. 1391-1396, 1974. 
[21] J. E. Goldthwait, "Slipping or recurrent dislocation of the patella: with the report of eleven cases," The Journal of Bone and Joint Surgery-American Volume, vol. 85, no. 12, p. 2489, 2003.

[22] E. E. Berg, S. L. Mason, and M. J. Lucas, "Patellar height ratios. A comparison of four measurement methods," American Journal of Sports Medicine, vol. 24, no. 2, pp. 218-221, 1996.

[23] C. A. Laurin, R. Dussault, and H. P. Levesque, "The tangential X-ray investigation of the patellofemoral joint: X-ray technique, diagnostic criteria and their interpretation," Clinical Orthopaedics and Related Research, no. 144, pp. 16-26, 1979.

[24] C. A. Laurin, H. P. Lévesque, R. Dussault, H. Labelle, and J. P. Peides, "The abnormal lateral patellofemoral angle. A diagnostic roentgenographic sign of recurrent patellar subluxation," The Journal of Bone and Joint Surgery. American Volume, vol. 60, no. 1, pp. 55-60, 1978.

[25] C. Thévenin-Lemoine, M. Ferrand, A. Courvoisier, J.-P. Damsin, H. D. Le Pointe, and R. Vialle, "Is the Caton-Deschamps index a valuable ratio to investigate patellar height in children?" The Journal of Bone and Joint Surgery-American Volume, vol. 93, no. 8, article e35, 2011.

[26] V. J. Avikainen, R. K. Nikku, and T. K. Seppanen-Lehmonen, "Adductor magnus tenodesis for patellar dislocation: technique and preliminary results," Clinical Orthopaedics and Related Research, no. 297, pp. 12-16, 1993.

[27] P. A. Kolowich, L. E. Paulos, T. D. Rosenberg, and S. Farnsworth, "Lateral release of the patella: indications and contraindications," The American Journal of Sports Medicine, vol. 18, no. 4, pp. 359-365, 1990.

[28] J. E. Goldthwait, "V. Permanent dislocation of the patella. The report of a case of twenty years' duration, successfully treated by transplantation of the patella tendons with the tubercle of the tibia," Annals of Surgery, vol. 29, no. 1, pp. 62-68, 1899.

[29] P. Sillanpää, V. M. Mattila, T. Visuri, H. Mäenpää, and H. Pihlajamäki, "Ligament reconstruction versus distal realignment for patellar dislocation," Clinical Orthopaedics and Related Research, vol. 466, no. 6, pp. 1475-1484, 2008.

[30] A. Panagopoulos, L. van Niekerk, and I. K. Triantafillopoulos, "MPFL reconstruction for recurrent patella dislocation: a new surgical technique and review of the literature," International Journal of Sports Medicine, vol. 29, no. 5, pp. 359-365, 2008.

[31] P. J. Sillanpää, H. M. Mäenpää, V. M. Mattila, T. Visuri, and H. Pihlajamäki, "A mini-invasive adductor magnus tendon transfer technique for medial patellofemoral ligament reconstruction: a technical note," Knee Surgery, Sports Traumatology, Arthroscopy, vol. 17, no. 5, pp. 508-512, 2009.

[32] E. Nomura, M. Inoue, and S. Kobayashi, "Long-term followup and knee osteoarthritis change after medial patellofemoral ligament reconstruction for recurrent patellar dislocation," The American Journal of Sports Medicine, vol. 35, no. 11, pp. 18511858, 2007.

[33] D. Drez Jr., T. B. Edwards, and C. S. Williams, "Results of medial patellofemoral ligament reconstruction in the treatment of patellar dislocation," Arthroscopy, vol. 17, no. 3, pp. 298-306, 2001.

[34] J. L. Ellera Gomes, "Medial patellofemoral ligament reconstruction with half width (hemi tendon) semitendinosus graft," Orthopedics, vol. 31, no. 4, pp. 322-326, 2008.

[35] R. M. Dopirak, R. N. Steensen, and P. B. Maurus, "The medial patellofemoral ligament," Orthopedics, vol. 31, no. 4, pp. 331-338, 2008.
[36] R. T. Burks and M. G. Luker, "Medial patellofemoral ligament reconstruction," Techniques in Orthopaedics, vol. 12, no. 3, pp. 185-191, 1997.

[37] T. Muneta, I. Sekiya, M. Tsuchiya, and K. Shinomiya, "A technique for reconstruction of the medial patellofemoral ligament," Clinical Orthopaedics and Related Research, no. 359, pp. 151-155, 1999.

[38] B. Fisher, J. Nyland, E. Brand, and B. Curtin, "Medial patellofemoral ligament reconstruction for recurrent patellar dislocation: a systematic review including rehabilitation and return-tosports efficacy," Arthroscopy, vol. 26, no. 10, pp. 1384-1394, 2010.

[39] M. Deie, M. Ochi, Y. Sumen, N. Adachi, K. Kobayashi, and M. Yasumoto, "A long-term follow-up study after medial patellofemoral ligament reconstruction using the transferred semitendinosus tendon for patellar dislocation," Knee Surgery, Sports Traumatology, Arthroscopy, vol. 13, no. 7, pp. 522-528, 2005.

[40] S. F. Fucentese, A. Von Roll, P. P. Koch, D. R. Epari, B. Fuchs, and P. B. Schottle, "The patella morphology in trochlear dysplasiaa comparative MRI study," Knee, vol. 13, no. 2, pp. 145-150, 2006.

[41] M. Jacobi, N. Reischl, M. Bergmann, S. Bouaicha, V. Djonov, and R. A. Magnussen, "Reconstruction of the medial patellofemoral ligament using the adductor magnus tendon: an anatomic study," Arthroscopy, vol. 28, no. 1, pp. 105-109, 2012.

[42] R. F. LaPrade, A. H. Engebretsen, T. V. Ly, S. Johansen, F. A. Wentorf, and L. Engebretsen, "The anatomy of the medial part of the knee," The Journal of Bone and Joint Surgery-American Volume, vol. 89, no. 9, pp. 2000-2010, 2007.

[43] W. Rauschning, L. O. Nordesjö, and B. Nordgren, "Isokinetic extension strength and pain before and after correction of recurrent patellar dislocation," Archives of Orthopaedic and Traumatic Surgery, vol. 102, no. 2, pp. 102-106, 1983. 


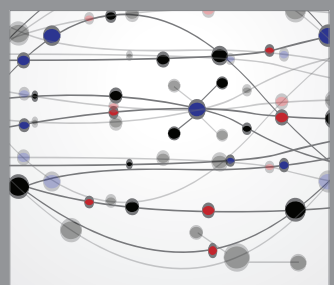

The Scientific World Journal
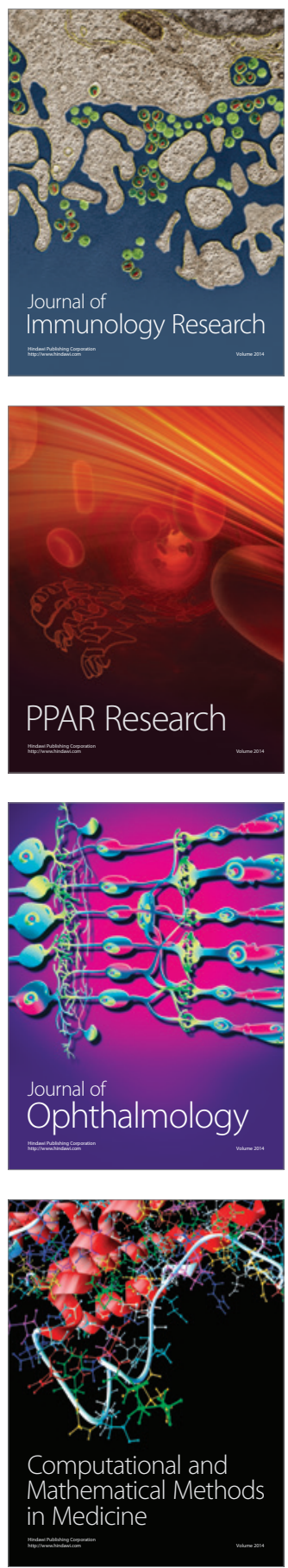

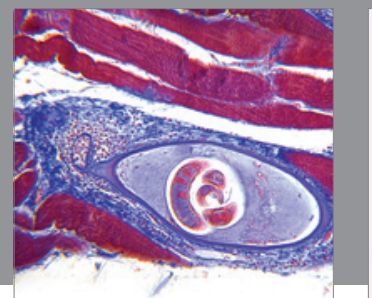

Gastroenterology

Research and Practice
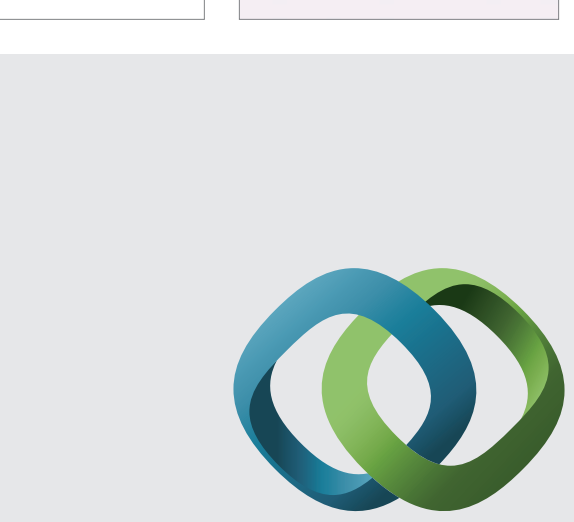

\section{Hindawi}

Submit your manuscripts at

http://www.hindawi.com
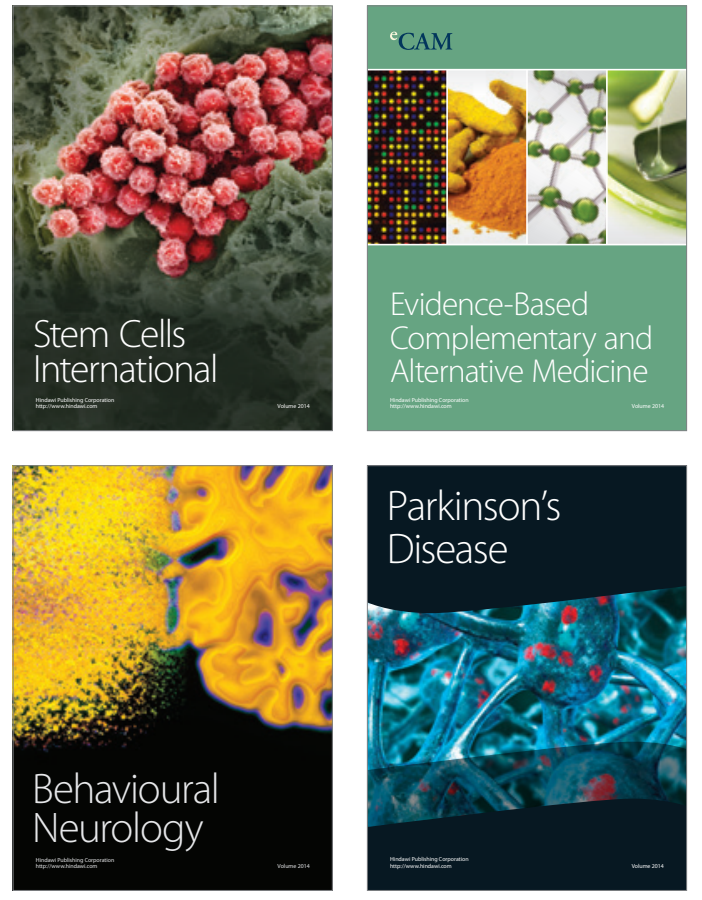
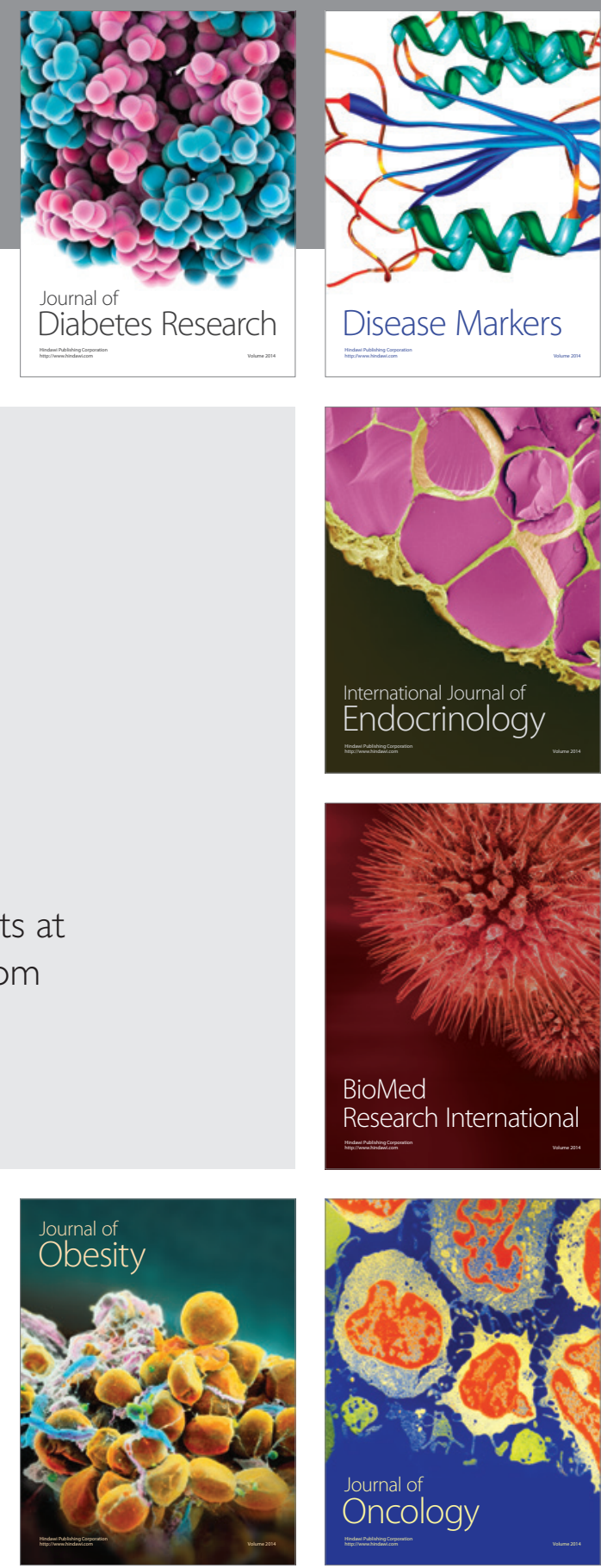

Disease Markers
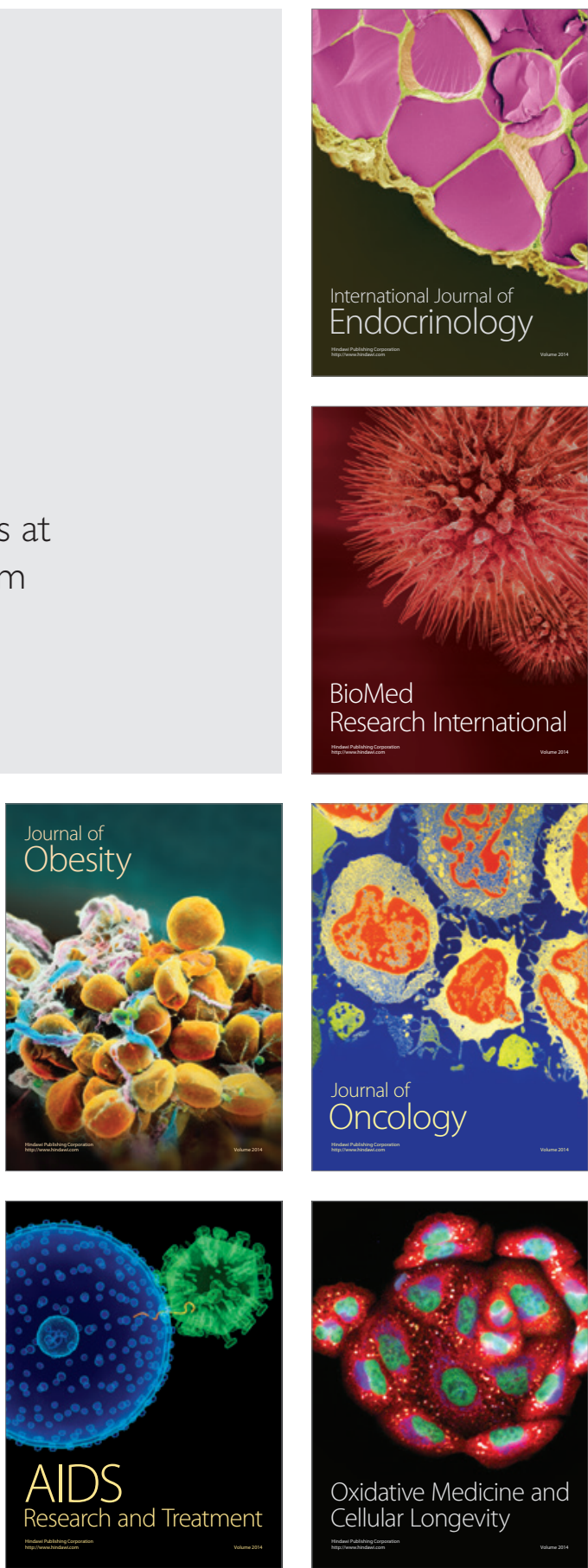